\title{
Cartographie de la croissance urbaine de Kinshasa (R.D. Congo) entre 1995 et 2005 par télédétection satellitaire à haute résolution
}

The mapping of the urban growth of Kinshasa (DRC) through high resolution remote sensing between 1995 and 2005

\section{Matthieu Kayembe Wa Kayembe, Mathieu De Maeyer et Eléonore Wolff}

\section{(2) OpenEdition Journals}

Édition électronique

URL : http://journals.openedition.org/belgeo/7349

DOI : $10.4000 /$ belgeo.7349

ISSN : 2294-9135

\section{Éditeur :}

National Committee of Geography of Belgium, Société Royale Belge de Géographie

Édition imprimée

Date de publication : 31 décembre 2009

Pagination : 439-456

ISSN : 1377-2368

Référence électronique

Matthieu Kayembe Wa Kayembe, Mathieu De Maeyer et Eléonore Wolff, « Cartographie de la croissance urbaine de Kinshasa (R.D. Congo) entre 1995 et 2005 par télédétection satellitaire à haute résolution », Belgeo [En ligne], 3-4 | 2009, mis en ligne le 17 mars 2013, consulté le 10 décembre 2020 URL : http://journals.openedition.org/belgeo/7349; DOI : https://doi.org/10.4000/belgeo.7349 


\title{
Cartographie de la croissance urbaine de Kinshasa (R.D. Congo) entre 1995 et 2005 par télédétection satellitaire à haute résolution
}

\author{
Matthieu Kayembe Wa Kayembe \\ Université de Lubumbashi \\ Mathieu De Maeyer, Eléonore Wolff \\ Université Libre de Bruxelles
}

RÉSUMÉ

La croissance spatiale de Kinshasa (RDC) est cartographiée par classification des espaces bâtis au départ d'images satellitaires SPOT datant de 1995 et 2005. D'après les résultats, la croissance de la ville est moins rapide que l'évolution démographique; elle s'effectue désormais dans les espaces interstitiels en dépit de leurs fortes pentes et d'un certain éloignement des principales voies de communication (au delà d'1 km).

MOTS-CLÉS: urbain, Afrique, télédétection

\begin{abstract}
THE MAPPING OF THE URBAN GROWTH OF KINSHASA (DRC) THROUGH HIGH RESOLUTION REMOTE SENSING BETWEEN 1995 AND 2005

The urban growth of Kinshasa (DRC) is mapped by classification of built-up areas using SPOT images dating from 1995 and 2005. From the results, the city growth is slower than the population growth; it is taking place within interstitial areas despite their steep slopes and their distance $(1 \mathrm{~km})$ from the main communication axes.
\end{abstract}

KEY WORDS: urban, Africa, remote sensing 


\section{INTRODUCTION}

$\mathrm{D}$ epuis les années 1950, la plupart des villes africaines ont connu des taux de croissance annuels exceptionnels (de 5 à 9\% pour la période de 1950 à 1990) soutenus par la forte croissance naturelle de la population urbaine et l'exode rural (Dubresson et al., 1998). Cette croissance conduit à des transformations spatiales rapides de l'espace urbain. Les villes s'étendent sur les espaces périurbains, jadis voués à l'activité agricole, et se densifient dans les quartiers centraux; Kinshasa n'échappe pas à ce constat (Delbart et al., 2002). Son emprise spatiale récente reste mal connue.

En effet, le document cartographique le plus récent illustrant les limites de la zone urbaine est l'atlas de Kinshasa (Flouriot et al., 1975), qui permet de comprendre l'organisation urbaine des années 1970.

Le recours à la télédétection satellitaire constitue une source de données utile étant donné l'extension considérable de la ville et l'absence de documents cartographiques actualisés. Cette étude a pour objectif de cartographier et de quantifier la croissance urbaine entre 1995 et 2005 en utilisant une série temporelle d'images satellitaires à haute résolution.
Suivre l'évolution urbaine de Kinshasa a toujours été la préoccupation des géographes et aménageurs. Ces derniers ont longtemps privilégié l'approche cartographique pour retracer l'extension spatiale de la ville. Cette dernière consiste à rassembler de documents cartographiques anciens et à les comparer (Fouriot, 1975; Pain, 1978). Flouriot (1975) a également combiné l'approche cartographique avec les enquêtes auprès des ménages pour suivre la croissance de l'habitat replacée dans le temps long.

Le développement de l'informatique et des techniques de la télédétection et des SIG a fait évoluer les méthodes. A Kinshasa, Tshibangu et al. (1997) ont intégré dans un SIG la carte de végétation dessinée par Compere en 1960 grâce à l'interprétation de photographies aériennes et les interprétations des images satellitaires réalisées par Wilmet en 1982 et Nseka en 1987 pour quantifier l'extension urbaine. Delbart et al. (2002) ont évalué l'extension de la ville à partir d'une carte existante (de 1969) et l'extension du bâti en 1995 observée sur une image SPOT (de 1995).

\section{ZONE D'ÉTUDE}

La ville-province de Kinshasa, située entre $4^{\circ}$ et $5^{\circ}$ de latitude sud et entre 15 et $16^{\circ}$ de longitude est, est la plus grande ville de la République Démocratique du Congo. Elle couvre une superficie de 9965 km² (De Saint Moulin, 2005), dont environ $600 \mathrm{~km}^{2}$ seulement seraient urbanisés. La ville comptait 400000 habitants en 1960 et aurait atteint plus de six millions d'habitants en 2008; le taux de croissance annuel moyen entre 1960 et 2003 serait de l'ordre de 6,8\% (Lelo Nzuzi, 2008).
Kinshasa s'est développée dans la plaine en bordure du fleuve Congo. La plaine, située à 300 mètres d'altitude, s'étend sur environs $200 \mathrm{~km}^{2}$. C'est la zone la plus industrialisée, la plus densément et anciennement habitée; on l'appelle communément la ville «basse». Après l'indépendance en 1960, la ville s'est étendue sur le complexe collinaire entourant la ville basse et culminant environ à $600 \mathrm{~m}$ d'altitude; cette zone est essentiellement occupée par des quartiers spontanés. 


\section{DONNÉES}

Les trois images SPOT utilisées datent respectivement des 31 mars 1995, 10 avril 2000 et 1 juillet 2005. Elles sont enregistrées en panchromatique et multispectral. Elles sont d'une mauvaise qualité radiométrique. L'image de 1995 a une couverture nuageuse de $7 \%$ en multispectral et de $5 \%$ en panchromatique. Les images panchromatique et multispectrale de 2000 présentent une importante zone de brume au sud-ouest de la ville, tandis que celle de 2005 comprend $6 \%$ de couverture nuageuse en mode multispectral et $10 \%$ en mode panchromatique. Ces nuages et brumes témoignent de la difficulté d'obtenir une image sans nuage pour une zone de climat subéquatorial (chaud et humide avec une saison des pluies de 8 mois).

D'autres données ont été collectées, numérisées et si nécessaire géoréférencées pour analyser la croissance urbaine de la ville. II s'agit des cartes anciennes permettant de replacer la croissance de Kinshasa dans le temps long, de données de population, du relief et des axes de communication.

En plus de la carte de la dynamique de l'habitat de l'Atlas de Kinshasa (Flouriot, 1975), la carte «District urbain de Léopoldville au 1/60 000» présente le périmètre urbain en 1920. Le Plan de Léopoldville (carte au 1/15 500 éditée par la librairie congolaise Léopoldville) donne la limite de la ville en 1954. La carte «Plan des communes de Kinshasa et ses environs» au 1/20 000 éditée en 1959 par I'Institut Géographique du Zaïre porte le tracé des limites communales de la zone urbanisée en 1959. La carte "Ville de Kinshasa-zones de santé» (carte au 1/20 000 éditée en 1969 et révisée en 1997 d'après le fond de base du Plan de Kinshasa, édition provisoire, publiée par
I'Institut Géographique du Zaïre porte le tracé des limites communales de la zone urbanisée en 1969. Tous ces documents sont complètement dépassés par la situation actuelle (Delbart et al., 2002; Renard et al., 1997) et méritent d'être actualisés. Les données de population utilisées souffrent à la fois de la qualité et de la fiabilité dans un pays où les bureaux de l'Etat civil sont caractérisés par un fonctionnement défaillant et où le recensement général des populations n'est pas régulièrement organisé. A l'exception des effectifs de populations de 1970 et de 1984 issus de recensements, les autres sont des simples projections de I'Institut National de la Statistique.

Les cartes de couverture cartographique à l'échelle de 1/10 000 par l'Institut Géographique du Congo Belge (IGCB) datant de 1958 et couvrant la ville de Kinshasa ont été scannées. Les courbes de niveau à une équidistance de $5 \mathrm{~m}$ ont été digitalisées, corrigées et interpolées (IGEAT/ULB) par la technique du spline pour produire un modèle numérique de terrain et en dériver la pente.

Une partie des routes (au nord de la ville et l'extrême est, après l'aéroport) a été digitalisée à partir de la bande panchromatique de l'image SPOT du 10 avril 2000 et du plan de la ville de Kinshasa imprimé à l'échelle de 1/10 000 en mars 1970 par l'Institut Géographique du Congo. Les routes de l'ouest et du sud ont été mesurées au DGPS et corrigées par le logiciel Pathfinder. Le chemin de fer a été digitalisé à partir du plan de la ville de Kinshasa imprimé à l'échelle de 1/ 10000 en mars 1970 par l'Institut Géographique du Congo. La partie du sud a été complétée par digitalisation sur l'image SPOT panchromatique du 10 avril 2000. 


\section{MÉTHODOLOGIE(1)}

Deux approches de détection des changements existent. Les méthodes de comparaison «image-image» supposent une normalisation radiométrique; cette normalisation est délicate à mettre en oeuvre sur des données de saison différentes et de qualité radiométrique aussi variable (Singh, 1986; Alphan, 2003; Coppin et al., 2004; Yuan et al., 2005). En outre, elles ne permettent pas d'identifier la nature des changements. Les méthodes de comparaison post-classification comparent les classifications de l'occupation du sol produites indépendamment aux différentes dates (Gupta et al., 1985). Ce dernier groupe de méthodes est moins sensible aux différences de saison; elles identifient la nature du changement mais sont sensibles aux erreurs de classification. Pour détecter les changements, les classifications sont comparées deux à deux. De cette comparaison, on dérive la carte des changements qui permet de les localiser, ainsi que la matrice des changements qui reprend le nombre de pixels ayant changé ou non de classes entre les deux dates et permet de quantifier les changements.

CORRECTION GÉOMÉTRIQUE, RECOUVREMENT ET DÉCOUPAGE DES IMAGES

Pour détecter les changements, il est indispensable que les images SPOT soient correctement calées du point de vue géométrique. Les corrections géométriques effectuées ne tiennent pas comp- te du relief car les décalages engendrés sont peu importants par rapport à la résolution spatiale des images utilisées (SPOT à 10 et $20 \mathrm{~m}$ ).

C'est pourquoi l'image panchromatique la plus récente a été corrigée par rapport à une image de résolution plus fine. II s'agit d'une image IKONOS panchromatique datant de 2002 d'une résolution d'1 m, corrigée elle-même avec des points de contrôle mesurés en mode absolu avec un GPS de marque Garmin GP60. Des erreurs quadratiques moyennes de 9,46 m sur la partie collinaire et 4,14 m sur la plaine ont été obtenues.

Ensuite, toutes les autres images SPOT (en mode panchromatique et multispectral) ont été corrigées sur l'image SPOT panchromatique corrigée avec une fonction polynomiale du premier ordre et par la méthode du plus proche voisin. Elles sont toutes projetées sur l'ellipsoïde WGS 84 en coordonnées UTM, zone 33 sud.

Les corrections géométriques aboutissent à des erreurs quadratiques moyennes inférieures à la taille d'un pixel avec 29 à 35 points de calage (tableau 1), ce qui est acceptable d'après MollerJensen (1990) et convient à une étude de détection des changements.

Toutes les images SPOT ne présentent pas la même extension spatiale. De plus, leur taille étant de $60 \mathrm{~km}$ de côté, celle-ci est plus large que l'extension de la ville de Kinshasa. Les images de 1995 et de 2005 ont été découpées à la même extension. Celle de 2000 est sensiblement

\begin{tabular}{|l|c|c|c|c|c|c|}
\hline Images/cartes & $\begin{array}{l}\text { Résolution } \\
\text { (en m) }\end{array}$ & $\begin{array}{l}\text { Nbre de } \\
\text { points } \\
\text { de calage }\end{array}$ & $\begin{array}{l}\text { X RMS } \\
\text { (pixels) }\end{array}$ & $\begin{array}{l}\text { XRMS } \\
\text { (en m) }\end{array}$ & $\begin{array}{l}\text { Y RMS } \\
\text { (pixels) }\end{array}$ & $\begin{array}{l}\text { Y RMS } \\
\text { (en m) }\end{array}$ \\
\hline $\begin{array}{l}\text { Spot panchromatique } \\
1995\end{array}$ & 10 & 30 & 0,44 & 4,4 & 0,57 & 5,7 \\
\hline Spot multispectral 1995 & 20 & 34 & 0,49 & 9,8 & 0,54 & 10,8 \\
\hline $\begin{array}{l}\text { Spot panchromatique } \\
2000\end{array}$ & 10 & 29 & 0,87 & 8,7 & 0,55 & 5,5 \\
\hline Spot multispectral 2000 & 20 & 32 & 0,64 & 12,8 & 0,29 & 5,8 \\
\hline Spot multispectral 2005 & 20 & 29 & 0,53 & 10,6 & 0,44 & 8,8 \\
\hline
\end{tabular}

Tableau 1. Erreurs quadratiques moyennes après correction géométrique. 
décalée vers l'ouest mais couvre néanmoins l'essentiel de la zone bâtie.

\section{CLASSIFICATION DE L'OCCUPATION DU SOL}

Etant donné la qualité inégale des images SPOT et la forte texture du bâti, elles ont été classées selon une méthode supervisée et orientée-objet à l'aide du logiciel eCognition

\section{Légende}

La légende distingue 4 classes: le bâti, le non bâti (végétation et sols nus), l'eau et les nuages.

Certaines classes sont difficiles à discriminer à l'aide des seules caractéristiques spectrales, et ce particulièrement dans les pays de l'Afrique subsaharienne. Les confusions spectrales sont nombreuses: par exemple, les champs se confondent facilement avec le bâti. Les bâtiments industriels et services et les immeubles à fonction résidentielle présentent à certains endroits la même signature spectrale que le banc de sable et les surfaces brûlées. Pour surmonter ces problèmes, nous avons enrichi la description spectrale des régions de paramètres de texture (cf. ci-après «Choix des attributs»).

\section{Sélection des zones d'entraînement et de validation}

Les zones d'entraînement et de validation ont été choisies sur base d'une interprétation visuelle des images SPOT appuyée d'une consultation de Google Earth et du plan de la ville de Kinshasa édité par Kin Art et Aquaterra en 1997, en veillant aux changements dus aux différences de dates entre tous ces documents. 68 zones ont été sélectionnées sur les parties communes aux images SPOT. Pour assurer un contenu équivalent des classes à chaque date, seules des zones inchangées entre 1995 et 2005 ont été sélectionnées. L'échantillon a été divisé en 2, 34 zones pour l'entraînement et 34 pour la validation.

\section{Choix des attributs}

Les attributs utilisés lors des classifications ont été choisis sur base d'une interprétation visuelle. Les régions sont décrites du point de vue spectral par les moyennes dans chaque bande spectrale ainsi que le NDVI et du point de vue textural, par les écart-types sur les bandes verte et rouge ainsi que deux paramètres de texture d'Haralick (1973) que sont l'homogénéité et l'entropie sur la bande panchromatique.

\section{Segmentation et classification}

eCognition peut utiliser simultanément des données variées, des images panchromatiques et multispectrales ou encore des bases de données vectorielles et peut créer plusieurs niveaux de segmentation grâce à une approche hiérarchique.

L'algorithme de segmentation est le "multiresolution segmentation". Selon le "Definiens Developer 7 User Guide" (2007), cet algorithme fusionne les pixels en segments d'image en minimisant l'hétérogénéité moyenne et en maximisant leurs homogénéités respectives. II peut agir de même avec des segments d'image provenant d'une segmentation précédente. La procédure fusionne itérativement les pixels ou les segments aussi longtemps que le seuil maximal d'hétérogénéité n'est pas dépassé. L'homogénéité est définie comme une combinaison de propriétés spectrales et de forme. L'homogénéité spectrale est basée sur l'écart-type de la distribution de la couleur et l'homogénéité de forme est basée sur l'écart par rapport à une forme compacte ou lisse (Cantou et al., 2006). La procédure peut être influencée par le facteur d'échelle qui limite la taille des segments résultants. La segmentation a été effectuée sur l'image de 2005 sur les bandes spectrales du vert, du rouge et du proche infrarouge en leur accordant respectivement un poids de 2 , 1 et 1 . Le paramètre d'échelle a été choisi par essais et erreurs et fixé à 20 et le paramètre de forme à 0.1 ( 0.5 pour la compacité et 0.5 pour le lissage). 
L'algorithme de classification supervisée du plus proche voisin a été utilisé. II classe les régions en fonction de leur proximité statistique aux zones d'entraînement.

\section{Validation}

Les classifications sont évaluées par comparaison des 34 zones de validation au sein de matrices de confusion. Différents indices sont calculés pour évaluer la qualité des résultats (Richards, 1993):

- l'exactitude générale,

- le Kappa global,

- le Kappa par classe.

L'exactitude générale est bonne (> 80 \%) pour les différentes classifications obtenues (tableau 2). Le coefficient Kappa n'est acceptable que pour les classifications de 1995 (85\%) et de 2005 (92\%). La classification de 2000 présente un mauvais Kappa (64\%) causé par la brume qui recouvre le sud-ouest de la ville. Ce résultat ne sera dès lors pas utilisé par la suite.

Les visites de terrain approfondies menées de la fin janvier 2009 à la fin mars 2009 dans les zones d'extension de Kinshasa pour comprendre les facteurs d'urbanisation ont révélé l'existence de différentes confusions et d'omissions pour la classe «bâti». A titre d'exemple, en voici quelques unes pour l'image de 1995 et pour 2005. Elles sont localisées à la figure 1 et identifiées au tableau 3.

Ces erreurs ne seront pas prises en compte dans l'analyse des résultats.

Les résultats obtenus aux différentes dates sont généralisés en supprimant les polygones classés «bâti» dont la superficie est inférieure à $1 \mathrm{~km}^{2}$ ainsi que les inclusions inférieures à $1 \mathrm{~km}^{2}$ au sein de la tache urbaine.

La superposition des classes bâties à deux dates successives permet de cartographier l'évolution du bâti alors que la matrice des changements permet de la quantifier.

\begin{tabular}{|l|c|c|}
\hline Années & Exactitude générale (\%) & Coefficient Kappa (\%) \\
\hline 1995 & 93 & 85 \\
\hline 2000 & 82 & 64 \\
\hline 2005 & 96 & 92 \\
\hline
\end{tabular}

Tableau 2. Exactitude des classifications.

\begin{tabular}{|l|l|}
\hline & Erreurs de confusion en 1995 \\
\hline Zone 1 & pirogues à l'est de Limete industriel \\
\hline Zone 2 & lle de Mimosa \\
\hline Zone 3 & champs et sablière au sud-ouest dans la commune de Mont Ngafula \\
\hline & Erreurs de confusion en 2005 \\
\hline Zone 1 & sablière se trouvant derrière le camp CETA et les champs ou cultures maraîchères \\
\hline Zone 2 & terrain brûlé à l'est de la ville \\
\hline Zone 4 & lle de Mimosa avec ses grands rochers et une entreprise d'extraction des matériaux de construction \\
\hline Zone 5 & banc de sable à l'ouest \\
\hline & Erreurs d'omission en 2005 \\
\hline Zone 3 & résidences situées dans la zone de l'Etat major des forces armées congolaises (abondance de la végétation) \\
\hline
\end{tabular}

Tableau 3. Erreurs de confusion et d'omission pour la classe bâti en 1995 et en 2005. 

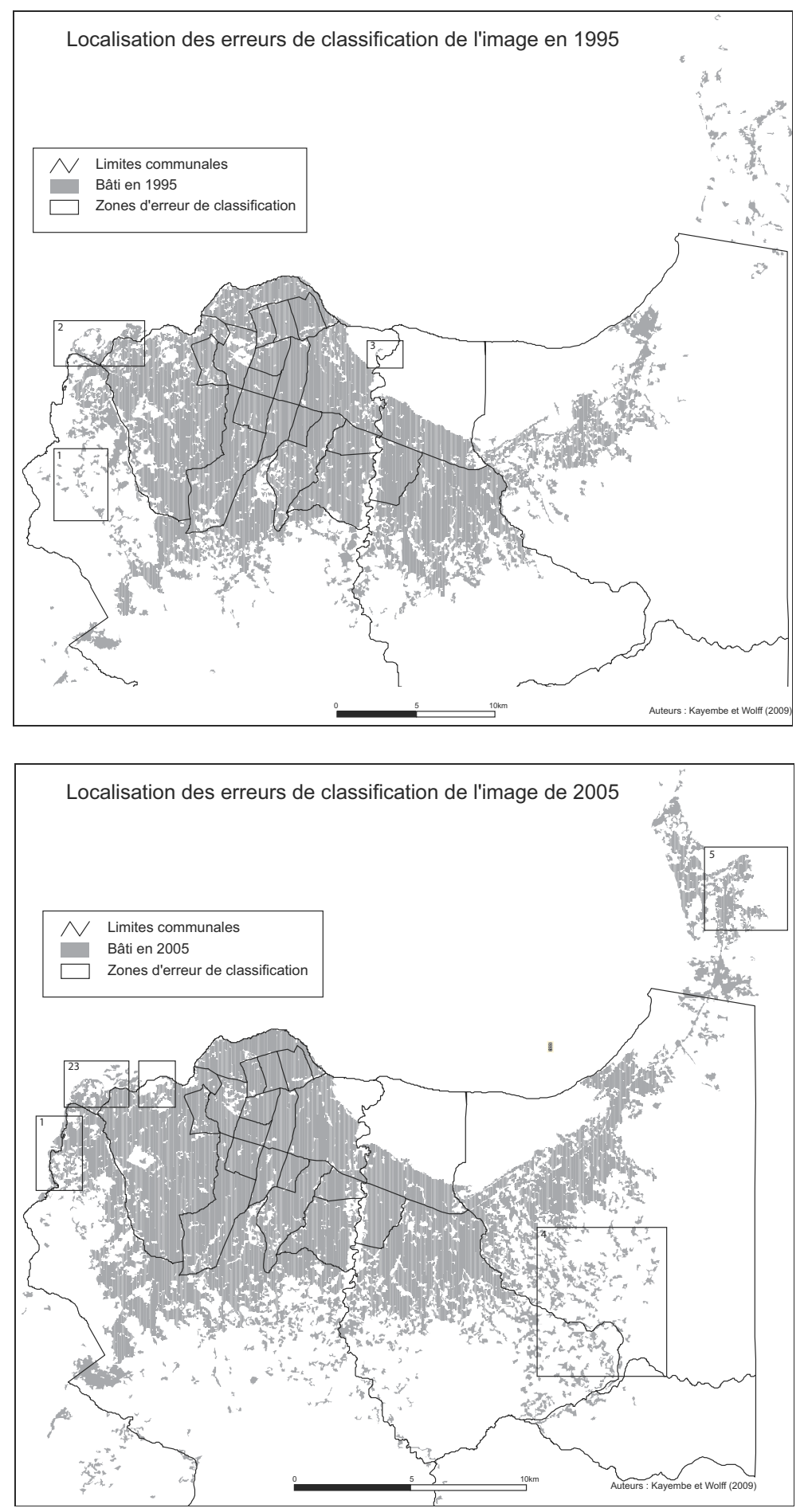

Figures 1a. - 1b. Localisation des erreurs de classification. Source: Delbart et Wolff, 2002 pour les limites des communes 
La localisation des changements a été analysée à l'aide:

- de visites de terrain approfondies menées de la fin janvier 2009 à la fin mars 2009 dans les zones d'extension de Kinshasa pour comprendre les facteurs d'urbanisation;

- du tracé des axes de transports routiers et de chemin de fer digitalisés;

- des cartes et des plans de la ville de Kinshasa;

- des données de population;

- du modèle numérique de terrain et de la carte des pentes.

La carte résultant de la comparaison des classifications d'occupation du sol de 1995 et 2005 montre que les extensions du bâti se concentrent vers le sud-ouest et vers le nord-est de Kinshasa (figure 2). Le sud-ouest a connu une extension spatiale le long des routes de Matadi et de Lutendele (zone 1). Ce processus a concerné les cités Benseke, Kimbondo, Sans fil, Matadi Mayo sur la route de Matadi et les cités Kimbala et Lutendele, Zamba, Mazanza sur la route de Lutendele. Les cités telles que Benseke et Kimbondo, Zamba et Kimbala se sont même rejointes en 2005.

A l'est, on observe un remplissage des espaces intersticiels (zone 2) et une extension (zone 3). En effet, tandis qu'à l'est de la rivière Ndjili, dans les quartiers Mpasa I, II et III, Mikonga et le camp Badara, les espaces vides se sont remplis, à l'extrême est, au delà de la rivière Ndjili (Cité Kinkole), le bâti s'est étendu.

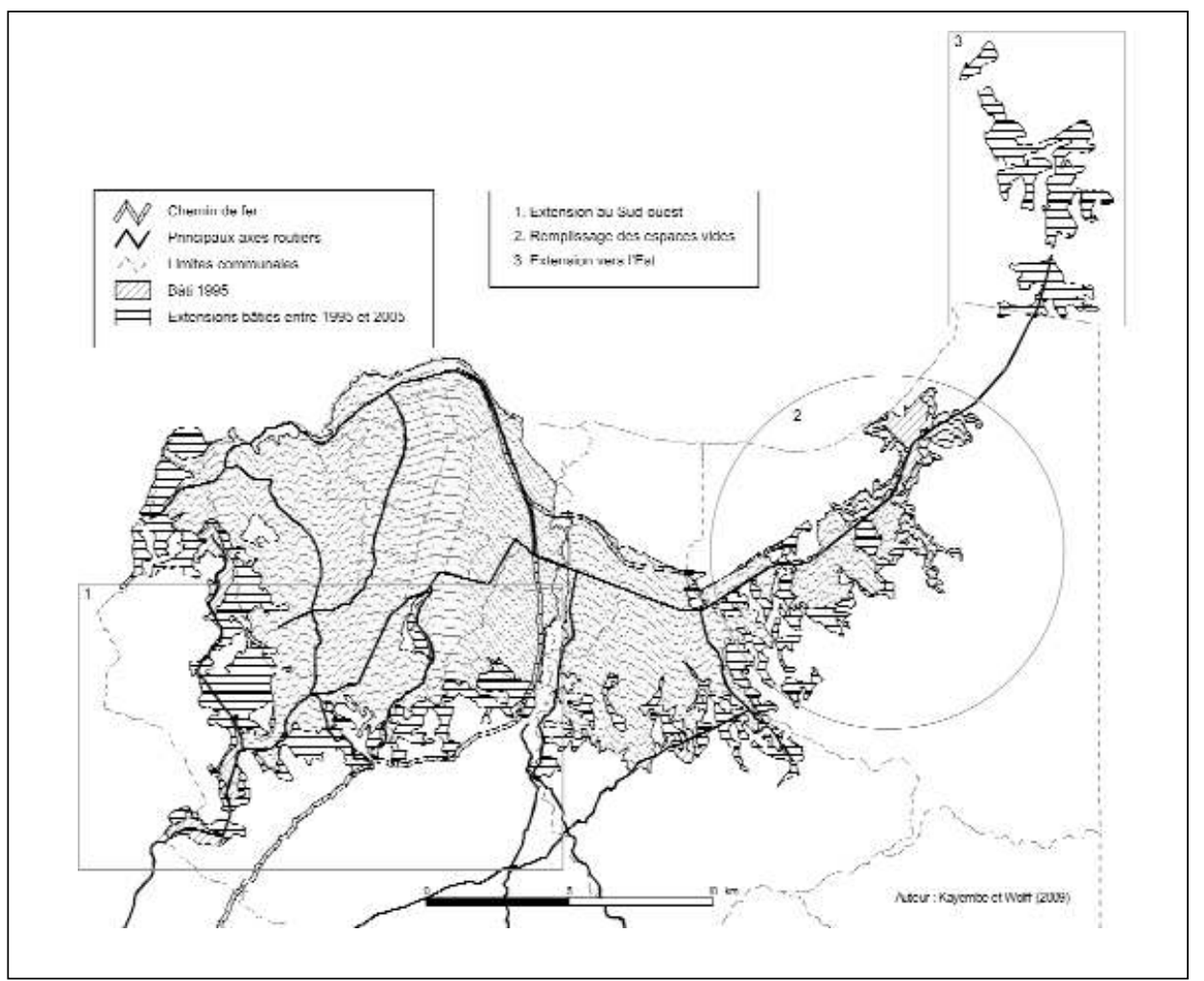

Figure 2. Cartographie de l'évolution de la tache urbaine entre 1995 et 2005. Sources: Images Spot KJ 3 096-358 du 31 mars 1995 et KJ 4 096-358 du 01 juillet 2005 
La croissance urbaine s'explique bien entendu par la croissance démographique. Sa localisation spatiale s'explique par deux facteurs géographiques principaux au delà de la simple distance au centre-ville, également centre d'emploi: le relief et les axes de communication.

CROISSANCE URBAINE ET CROISSANCE DÉMOGRAPHIQUE

Le tableau 4 reprend l'évolution de la population, de l'étendue de la ville de Kinshasa et de sa densité. Nos résultats, mis en perspective grâce aux sources bibliographiques, contredisent ou nuancent certains effectifs de population urbaine et superficies urbaines fréquemment utilisés (cf. zone d'étude). En 45 ans, la population passe de 400000 habitants à 7500000 en 2005; alors que la surface bâtie s'étend à 6800 ha en 1960 contre 43400 en 2005. La densité de la population triple entre 1960 et 2005 en passant d'environs 60 hab/ha en 1960 à 170 hab/ha en 2005; dans l'ensemble, la ville s'étend et se densifie.

La figure 3 montre que, par rapport à la

\begin{tabular}{|r|r|r|r|}
\hline Date & Population & $\begin{array}{c}\text { Surface } \\
\text { (ha) }\end{array}$ & $\begin{array}{r}\text { Densité } \\
\text { (hab/ha) }\end{array}$ \\
\hline 1960 & 400000 & 6800 & 59 \\
\hline 1967 & 901520 & 9470 & 95 \\
\hline 1969 & 1051000 & 12903 & 81 \\
\hline 1973 & 1323039 & 14600 & 91 \\
\hline 1975 & 1679091 & 17992 & 93 \\
\hline 1981 & 2567166 & 20160 & 127 \\
\hline 1984 & 2653558 & 26000 & 102 \\
\hline 1995 & 4719862 & 31007 & 152 \\
\hline 2000 & 6000000 & 39518 & 151 \\
\hline 2005 & 7500000 & 43414 & 173 \\
\hline
\end{tabular}

Tableau 4. Evolution de la population, de l'étendue de Kinshasa et de sa densité.

Sources: Lelo Nzuzi, 2008; Yebe Musieme Beni, 2004; Delbart et al., 2002; Matota Mbuila, 2001

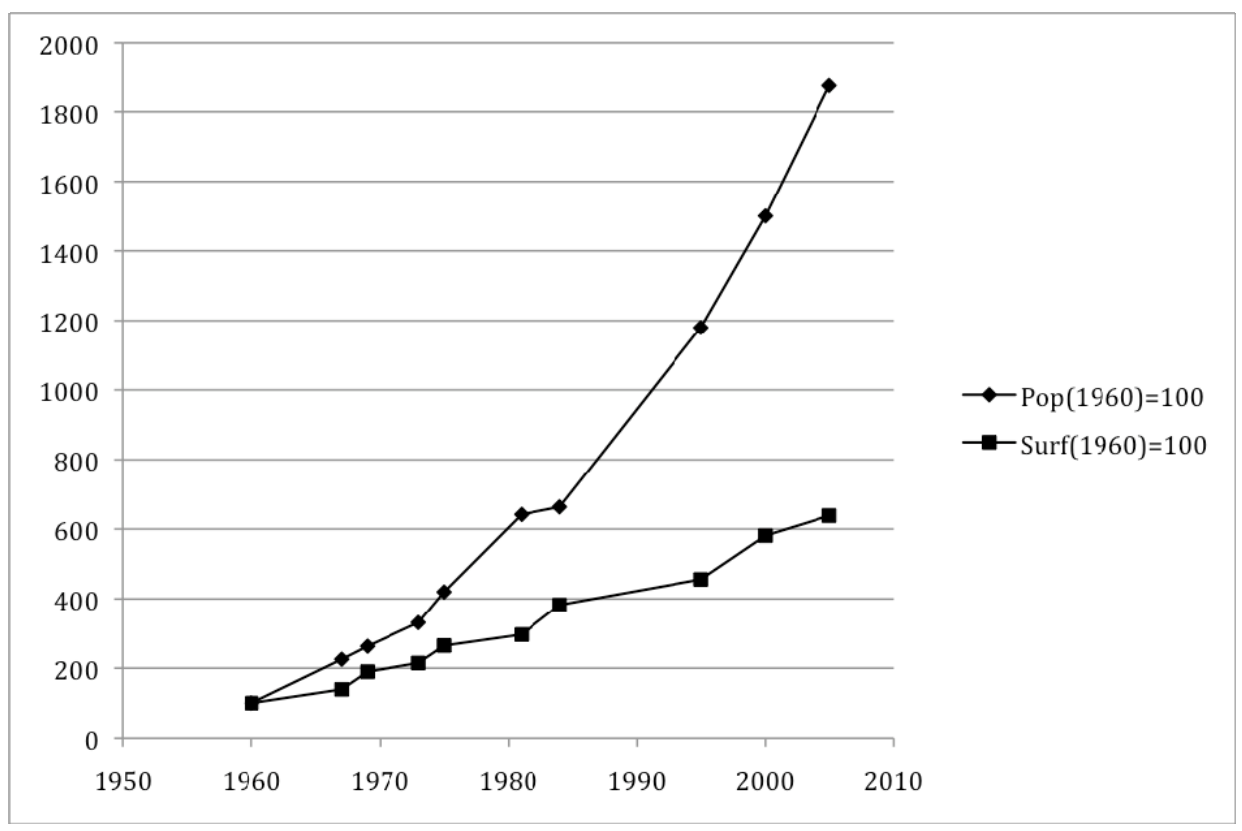

Figure 3. Croissance urbaine et croissance démographique.

Sources: Lelo Nzuzi, 2008; Yebe Musieme Beni, 2004; Delbart et al., 2002; Matota Mbuila, 2001 
situation de 1960 (indice 100), la population est à l'indice 1900 en 2005 et la surface bâtie à l'indice 600 .

Pour comparer les taux de croissance, on porte les données sur un graphique semilogarithmique (figure 4).

La figure 4 montre que l'évolution du taux de croissance de la surface bâtie et celui de la population entre 1960 et 2005. Le taux de croissance démographique est supérieur à celui de l'extension de la ville. Le taux de croissance moyen de la population sur la période 1960-2005 est de $6,73 \%$, alors que celui de la surface bâtie est de 4,21\%. En appliquant ce taux de croissance à la surface bâtie de 2005 pour calculer l'extension de la ville en 2009, on n'obtient pas les $600 \mathrm{~km}^{2}$ régulièrement cités, mais seulement environ $510 \mathrm{~km}^{2}$.
En analysant les pentes, on peut comparer les taux de croissance; ceux-ci semblent tous deux fléchir vers 1981 (tableau 5). Ce résultat confirme les observations de Bruneau (1994).

La première période couvre les 20 ans après l'indépendance (jusqu'en 1981). Elle est caractérisée par une croissance démographique très forte et une extension spatiale rapide. Au cours de la deuxième période, le taux de croissance démographique ralentit très nettement, même si ce dernier reste élevé, passant de 9,26\% entre 1960 et 1981 à 5,07 entre 1981 et 2005. L'extension spatiale croît à un rythme moins soutenu et passe de $5,31 \%$ entre 1960 et 1981 à 3,25 \% entre 1981 et 2005.

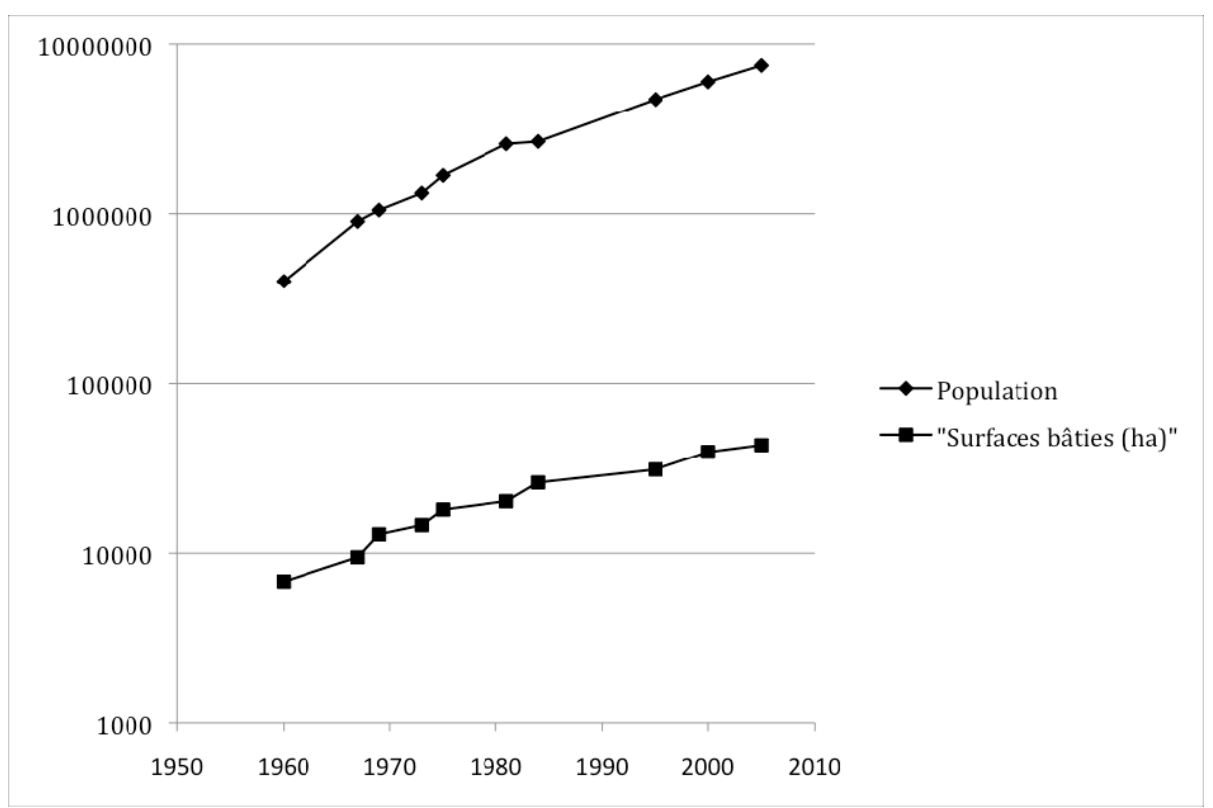

Figure 4. Graphique semi-logarithmique de croissance urbaine et de la croissance démographique.

Sources: Lelo Nzuzi, 2008; Yebe Musieme Beni, 2004; Delbart et al., 2002; Matota Mbuila, 2001

\begin{tabular}{|l|c|c|}
\hline Taux de croissance & démographique & urbaine \\
\hline $1960-1981$ & $9,26 \%$ & $5,31 \%$ \\
\hline $1981-2005$ & $5,07 \%$ & $3,25 \%$ \\
\hline
\end{tabular}

Tableau 5. Taux de croissance démographique et urbain. 


\section{CROISSANCE URBAINE ET RELIEF}

D'après l'observation des cartes et des plans existants (figure 5), l'extension vers Kinkole confirme les phases qui ont caractérisé le développement spatial de Kinshasa depuis sa création. Née à l'ouest vers la baie de Ngaliema, la ville a connu ses premiers développements vers l'est avec la naissance des anciennes cités (Kinshasa, Barumbu, Lingwala, Kintambo) et la fusion de Gombe (jadis Kalina) et Leo-Est vers la fin des années 1920. Depuis lors la ville a progressé vers le sud de la ville actuelle (Kalina+LeoEst) avec la naissance des nouvelles cités (Kasa Vubu et Ngiri Ngiri) entre les années 1930 et 1940. C'est au courant des années 1950 que la ville a repris la direction de l'est depuis la fusion de
Kalina (Léo Ouest) avec Léo Est. En comparant le périmètre urbain de 1959, on constate que la ville s'est beaucoup plus étendue au sud et sud-ouest qu'à l'est, au delà des communes de Kimbanseke et de Masina. En comparant cette croissance à la carte de l'altitude et des formes du relief, on observe que Kinshasa s'est d'abord étendue dans la plaine correspondant à l'extension ancienne du pool Malebo et ce jusqu'à l'indépendance en 1960 (figure 7); les autorités coloniales interdisaient strictement les constructions sur les collines en l'absence d'un aménagement particulier. Après l'indépendance, la ville s'est étendue vers le sud-ouest sur les plateaux et vers l'est dans la plaine. Pour la période 1995-2005, la croissance a continué dans les mêmes directions.

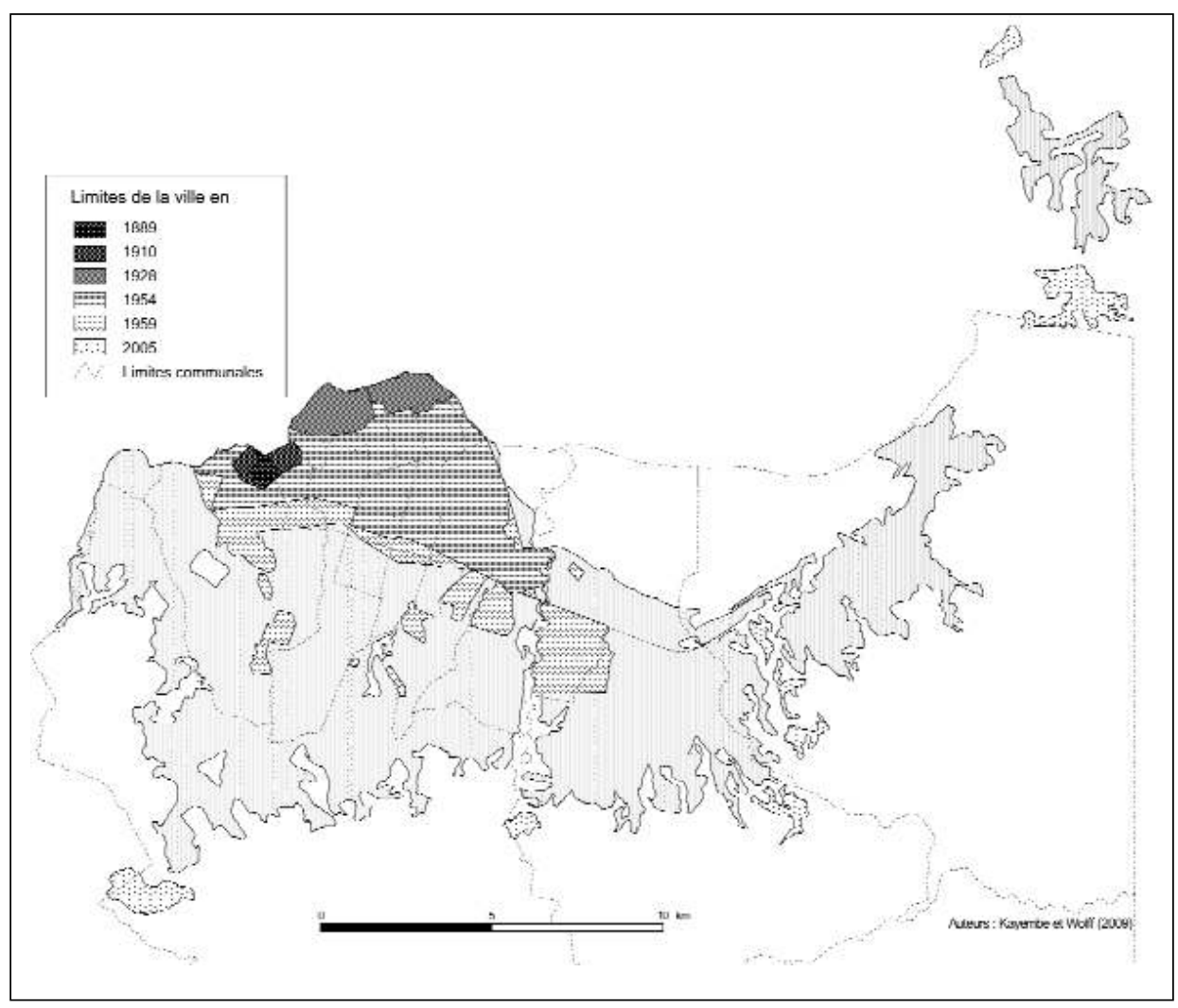

Figure 5. Croissance urbaine de Kinshasa de 1889 à 2005.

Sources: Cartes historiques collectées par Johan Lagae, Département d'Architecture et d'Urbanisme, Université de Gand 


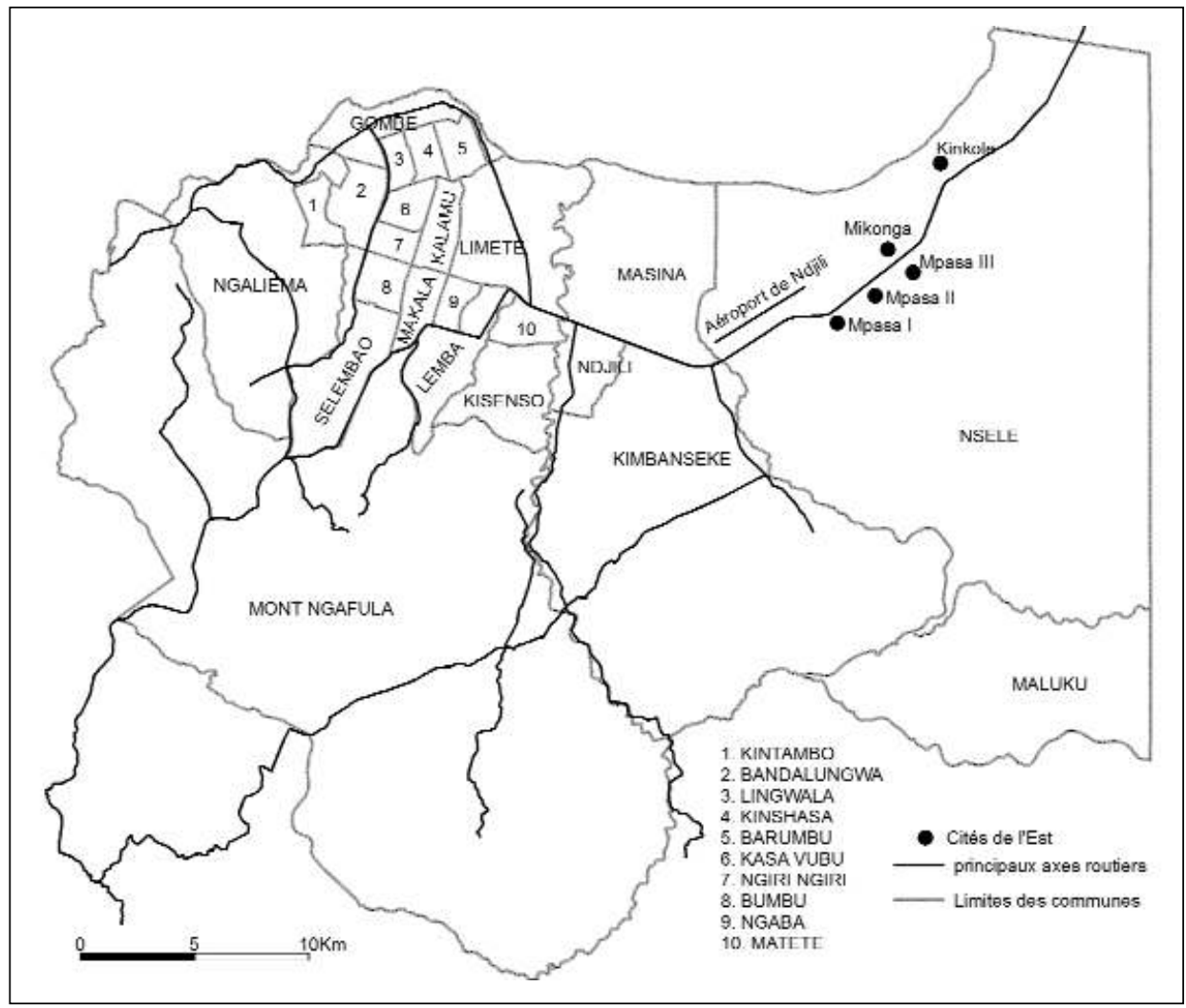

Figure 6. Communes et cités à l'est de Kinshasa.

Vers l'est, elle s'allonge en ruban dans la plaine qui est ici plus étroite (Biyeye, 1997). En effet, l'extension derrière les quartiers Mpasa I, II, III et Mikonga n'a pas eu lieu en raison des fortes pentes; c'est ainsi que l'extension urbaine s'est déplacée au-delà de la Cité Kinkole.

A l'ouest de la rivière Ndjili, les superficies planes des plateaux du sud étant construites, l'urbanisation s'étend sur les fortes pentes (figure 8); ces dernières sont impropres à l'implantation humaine en l'absence d'un aménagement approprié. En effet, ces zones de fortes pentes sont soumises à un risque important d'érosion dès qu'elles sont mises à nu (Van Caillie, 1990, 1997). De plus, les plaines en aval de ces fortes pentes sont touchées par des inondations à cause de l'ensablement. Ces zones de fortes pentes abritent des populations défavorisées. Dans le futur, l'urbanisation continuera à se localiser sur les zones de forte pente comme c'est déjà le cas dans beaucoup d'endroits sur les flancs de collines (quartiers Camping, Kindele, etc.). Une analyse quantitative (par recouvrement statistique) des pentes par rapport à la croissance urbaine a été réalisée en utilisant trois classes de pentes (Van Caillie, 1997): faible (<10\%) présentant des faibles risques d'érosion; moyenne (10-15\%) dans lesquelles sont susceptibles de se déclencher des phénomènes d'érosion et forte (>15\%) présentant de forts risques d'érosion. Cette analyse a été réalisée uniquement sur la zone couverte par le MNT (cf. figure 8), ce qui représente environ $30 \%$ de l'extension urbaine totale entre 1995 et 2005. 


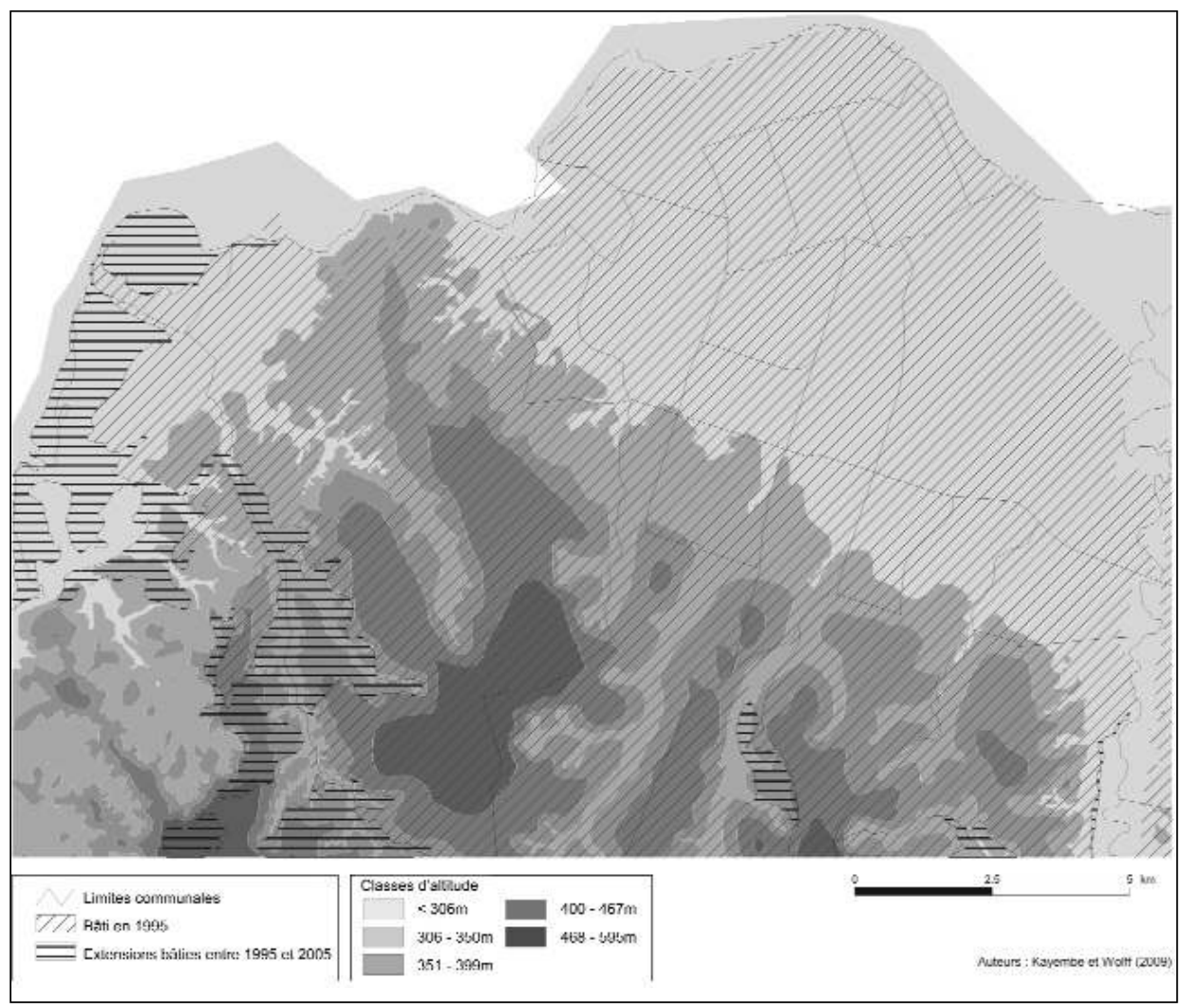

Figure 7. Urbanisation et altitude.

Source: Mathieu De Maeyer IGEAT/ULB pour le MNT, inédit

Les résultats (tableau 6) montrent que plus de $50 \%$ de la croissance est localisée sur des pentes faibles, tandis qu'environ $30 \%$ de la croissance a eu lieu sur les fortes pentes. La population a logiquement tendance à se localiser préférentiellement sur des pentes faibles quand cette possibilité existe. Néanmoins, plus de $30 \%$ de l'extension urbaine a lieu sur des pentes supérieures à $15 \%$, généralement dans des espaces laissés vacants par les vagues d'urbanisation précédentes car trop accidentés. Cela signifie que les personnes s'installent là où elles peuvent, notamment dans les espaces interstitiels et même sur des fortes pentes.

Toutefois ces jugements ne sont valables que pour la zone collinaire du sud-ouest couverte par le MNT. En ce qui concerne l'extension à l'est, il est fort probable qu'elle ait lieu quasi exclusivement sur des pentes faibles.

\begin{tabular}{|c|c|c|c|}
\hline Classe & Pente (\%) & $\begin{array}{c}\text { Extension } \\
\text { urbaine }\left(\mathrm{km}^{2}\right)\end{array}$ & $\%$ \\
\hline 1 & $<10$ & 15,41 & 53,2 \\
\hline 2 & $10-15$ & 4,53 & 15,6 \\
\hline 3 & $>15$ & 9,01 & 30,1 \\
\hline Total & & $\mathbf{2 8 , 9 5}$ & $\mathbf{1 0 0}$ \\
\hline
\end{tabular}

Tableau 6. Classe des pentes et extension urbaine entre 1995 et 2005. 


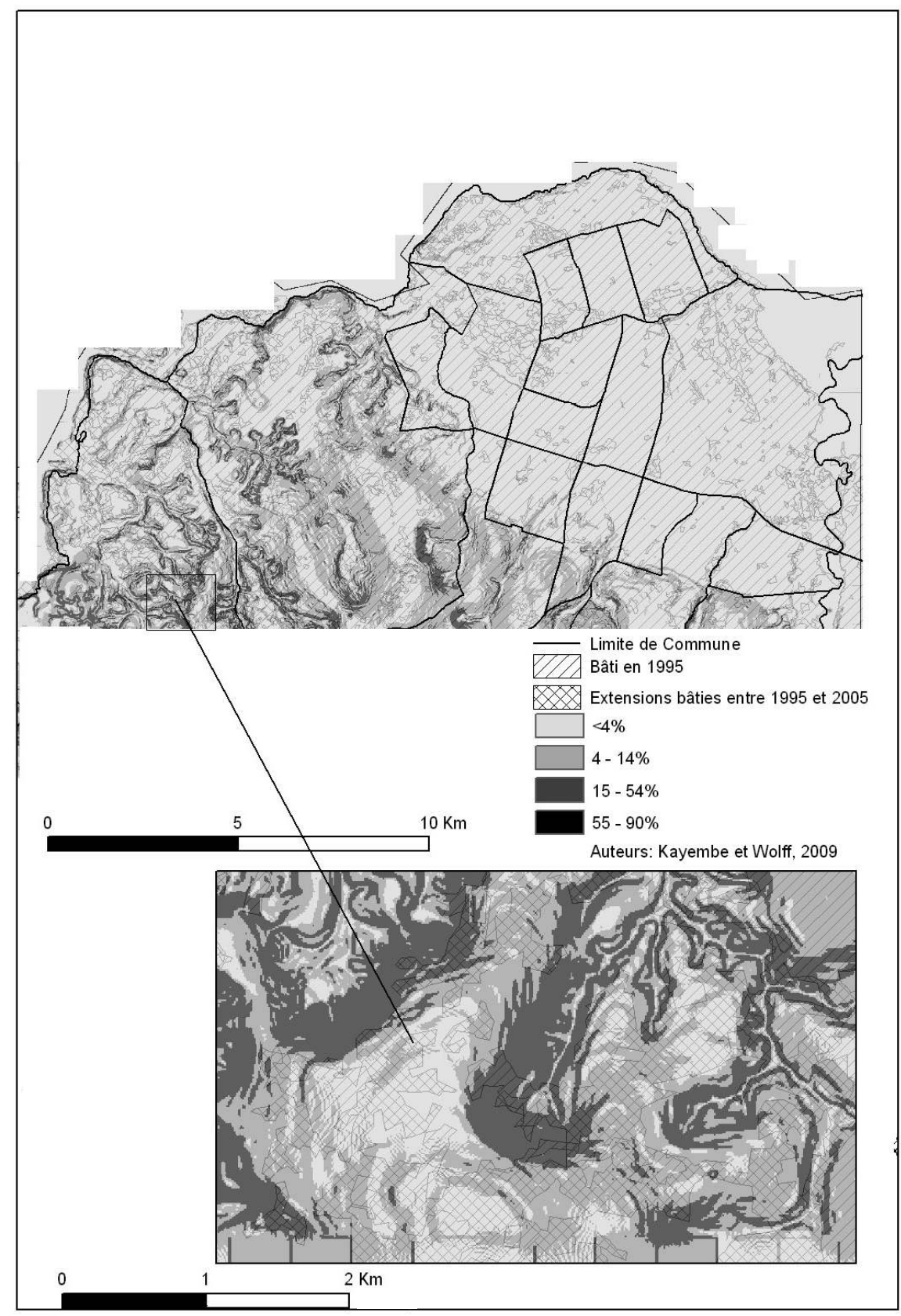

Figure 8. Croissance urbaine et pente.

Sources: Mathieu de Maeyer, IGEAT/ULB pour la carte des pentes, inédit 


\section{CROISSANCE URBAINE ET AXES DE COMMUNICATION}

La figure 2 montre que la croissance urbaine est plus influencée par les axes routiers que par le chemin de fer. Si avant l'indépendance le chemin de fer a joué un rôle dans la localisation des zones industrielles, le chemin de fer urbain ne s'étant pas développé n'a pas facilité l'accès au centre ville en tant que centre d'emploi et dès lors, n'a pas polarisé la croissance urbaine. Les routes de Matadi et du Bandundu, de même que les routes vers la chute de la Lukaya et vers le lac MaVallée, correspondent aux axes de la croissance urbaine actuelle.

Malgré le rôle joué par les axes routiers dans la croissance urbaine, il existe des quartiers récents qui se développent loin du centre ville et loin de ces axes. En effet, une zone d'extension urbaine a été observée depuis les années 1990 au sud et à l'est du campus où logent les professeurs de l'Université de Kinshasa. II s'agit du quartier Cogelos au sud et des quartiers Tchad, Mandela, Département et Plateau à l'est. Ces quartiers se développent en l'absence de commodités urbaines. En effet, ils ne sont pas connectés pour la quasi-totalité au service d'adduction d'eau de la Régideso. La

\begin{tabular}{|c|c|c|}
\hline $\begin{array}{c}\text { Distance } \\
\text { (mètres) }\end{array}$ & $\begin{array}{c}\text { Extension } \\
\text { urbaine }\left(\mathrm{km}^{2}\right)\end{array}$ & $\%$ \\
\hline$<200$ & 11,48 & 11,73 \\
\hline $200-1000$ & 40,37 & 41,25 \\
\hline$>1000$ & 46,02 & 47,02 \\
\hline Total & 97,87 & 100 \\
\hline
\end{tabular}

population s'approvisionne aux sources aménagées (Mayi ya Libanga, mayi ya Niwa, Mayi ya Nzamba) ou à la borne fontaine. C'est le cas du quartier Mbiti. Là où la nappe est haute, la population creuse un puits. L'absence de commodités urbaines ne limite pas la croissance de Kinshasa. L'essentiel pour la population est d'avoir un logement.

Une analyse quantitative de la croissance urbaine par rapport aux axes de communications majeurs a été réalisée en calculant la superficie de l'extension urbaine comprise dans des zones tampons de différentes distances autour de ces axes (200, 500 et 1000 mètres). Cette analyse montre que $47 \%$ de la croissance a lieu à plus d'un kilomètre de ces axes principaux (tableau 7). Au-delà d'un kilomètre, l'avantage de la proximité de la grande route peut être considéré comme secondaire; les gens s'installent à proximité des quartiers existants, à une plus grande distance de la route, notamment dans les zones de fortes pentes (cf. p. 449 «Croissance urbaine et relief»). Entre 1995 et 2005, l'urbanisation progresse dans les espaces interstitiels laissés vacants, car peu propices à l'urbanisation. Les axes de communication majeurs ne constituent pas toujours un facteur prépondérant dans la croissance urbaine.

Tableau 7. Distance de l'extension urbaine entre 1995 et 2005 par rapport aux axes routiers principaux. 
En l'absence de carte récente de la ville de Kinshasa, et surtout de ses extensions, deux images satellitaires à haute résolution (SPOT) de 1995 et 2005 ont permis de cartographier et de quantifier la croissance urbaine de Kinshasa de façon rapide, peu coûteuse et fiable. La ville s'étend très rapidement sur ses marges essentiellement vers l'est et le sud-ouest le long des routes de Matadi et du Bandundu, permettant un accès en transport en commun vers le centre ville qui polarise l'essentiel des emplois urbains. Cependant, depuis le début des années 1990, des quartiers se développent loin du centre ville et des axes de transport (ex: quartier Cogelo, Tchad, Mandela, Département, Plateau); ils ne bénéficient pourtant d'aucune commodité urbaine. L'extension de la ville n'a pas épargné après 1960 des zones de fortes pentes impropres à l'implantation humaine en l'absence d'un aménagement particulier. Ces zones abritent des populations défavorisées.

En mesurant le taux de croissance moyen de la population et l'extension de la ville sur 45 ans (de 1960 à 2005), on a constaté qu'il est de 6,73\% contre 4,21\% pour celui de la superficie bâtie. Le taux de croissance moyen de la surface bâtie appliqué à la surface bâtie en 2005 pour calculer la superficie bâtie en 2009 a mis en évidence des erreurs parfois non négligeables avec les chiffres régulièrement cités.

Entre 1995 et 2005, d'une part $30 \%$ de la croissance urbaine s'est effectuée sur des pentes de plus de $15 \%$, soit présentant un risque important d'érosion, et d'autre part, près de $50 \%$ de la croissance urbaine s'effectue à plus d'un kilomètre des axes majeurs de communication, soit dans les espaces interstitiels peu accessibles.

\section{BIBLIOGRAPHIE}

- ALPHAN H. (2003), «Land use change and urbanization in Adana, Turkey", Land Degradation and Development, 14 (6), pp. 575-586.

- BIYEYE UNZOLA E. (1997), Urbanisation de Kinshasa: la politique de la partie extrême orientale, Mémoire en Urbanisme et Aménagement, Université Libre de Bruxelles, $85 \mathrm{p}$

- BRUNEAU J-C. (1995), «Crise et déclin de la croissance des villes au Zaïre - Une image actualisée», Revue Belge de Géographie, 119e année, $N^{\circ}$ spécial offert au professeur H. Nicolaï, pp. 103-114.

- CANTOU J. P., MAILLET G., FLAMANC D. \& BUISSART H. (2006), «Preparing the use of pleiades images for mapping purposes: preliminary assessments at ign-france», Topographic Mapping from Space, ISPRS Workshop Commission I, WGI/5 and WG I/6, page 6 .

- COPPIN P., JONCKHEERE I., NACKAERTS K., MUYS B. \& LAMBIN E. (2004), «Digital change detection methods in ecosystem monitoring: A review», International Journal of Remote Sensing, 25, 9, pp.1565-1596.
- DElbart V. \& WOLFF E. (2002), «Extension urbaine et densité de la population à Kinshasa: contribution de la télédétection satellitaire», BELGEO, 2, 1, pp. 45-59.

- DE SAINT MOULIN L. (2005), Atlas de l'organisation administrative de la République Démocratique du Congo, Ed. Centre d'Etudes Pour l'Action Sociale (CEPAS), Kinshasa, $235 \mathrm{p}$

- DUBRESSON A. \& RAISON J.-P. (1998), L'Afrique subsaharienne: une géographie du changement, Armand Colin, Paris, $248 \mathrm{p}$

- FLOURIOT J., DE MAXIMY R. \& PAIN M. (1975), Atlas de Kinshasa.

- GUPTA D.N \& MUNSHI M.K. (1985), «Urban change detection and land-use mapping of Delhi», International Journal of Remote Sensing, vol. 6, n³-4, pp. 529-534.

- HARALICK R.M., (1979), "Statistical and structural approaches to texture», Proceedings of the IEEE, vol. $67, \mathrm{n}^{\circ} 5$, pp. 786-804.

- LELO NZUZI F. (2008), Kinshasa, ville et environnement, L'Harmattan, Paris, 275 p.

- MATOTA MBUILA R. (2001), La dynamique de l'espace urbain de Ngiri-Ngiri et la pro- 
blématique d'équipements publics pour son fonctionnement, Mémoire de Licence, Facultés Catholiques de Kinshasa, 72 p.

- MOLLER-JENSEN L. (1990), «Knowledgebased classification of an urban area using texture an context information in LandSat-TM imagery», Photogrammetric Engineering and Remote Sensing, vol. 56, n6, pp. 899904.

- PAIN M. (1978), Kinshasa: Ecologie et organisation urbaines, Thèse de doctorat, Université de Toulouse le Mirail, 470 p. + Annexes.

- SINGH A. (1986), «Digital change detection techniques using remotely sensed data», International Journal of Remote Sensing, 10, pp. 989-1003.

- TSHIBANGU KWT., ENGELS P. \& MALAISSE F. (1997), «Evolution du couvert végétal de la région de Kinshasa (19601987)» Geo-Eco-Trop, 21, 1-4, pp. 95-103.

- VAN CAILLIE X.D (1997), «La carte des pentes (1/20 000) de la région des collines à
Kinshasa", Cahier ORSTOM, réseau érosion, bulletin 17: Erosion en montagnes semi-arides et méditerranéennes, pp. 198204.

- VAN CAILLIE X.D. (1990), «Erodabilité des terrains sableux du Zaïre et contrôle de l'érosion», Cahier ORSTOM, série Pédologie, vol. XXV, n¹-2, pp. 197-208.

- YEBE MUSIEME BENI (2004), L'impact des érosions sur l'habitat à Kisenso et les travaux de lutte anti-érosive par la population locale, Travail de fin de cycle, Unikin, Faculté des Sciences, Département des Sciences de la terre, Géographie, 63 p., inédit.

- YUAN F., SAWAYA K.E., LOEFFELHOLZ B.C. \& BAUER M.E. (2005), "Land cover classification and change analysis of the Twin Cities (Minnesota) Metropolitan Area by multitemporal Landsat remote sensing", Remote Sensing of Environnement, 98, 2 \& 3, pp. 317-328

(1) Le lecteur se reportera au Précis de télédétection de F. Bonn pour les concepts de base de télédétection et traitements d'image: BONN F., ROCHON G. (1992), Précis de télédétection, Vol. n¹: Principes et méthodes, Québec, PUQ/AUPELF, 477 p.

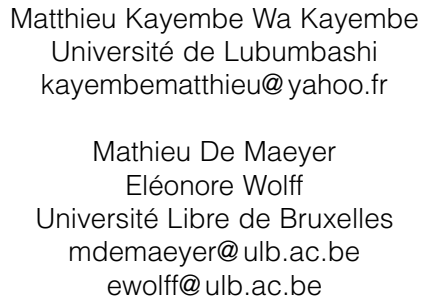

manuscrit déposé en septembre 2009; révisé en juin 2010 
